\title{
A Deep Learning Approach for Classification and Diagnosis of Parkinson's Disease
}

\section{Monika Jyotiyana}

Central University of Rajasthan

Nishtha Kesswani

Central University of Rajasthan

Munish Kumar ( $\square$ munishcse@gmail.com )

Maharaja Ranjit Singh Punjab Technical University https://orcid.org/0000-0003-0115-1620

\section{Research Article}

Keywords: Parkinson's Disease, Deep Neural Networks, Deep Learning, Classification

Posted Date: June 16th, 2021

DOI: https://doi.org/10.21203/rs.3.rs-254647/v1

License: (1) This work is licensed under a Creative Commons Attribution 4.0 International License. Read Full License 


\title{
A Deep Learning Approach for Classification and Diagnosis of Parkinson's Disease
}

\author{
Monika Jyotiyana ${ }^{1}$, Nishtha Kesswani ${ }^{1}$, Munish Kumar ${ }^{2(\text { Corresponding Author) }}$ \\ ${ }^{1}$ Central University of Rajasthan, Rajasthan, India \\ ${ }^{2}$ Maharaja Ranjit Singh Punjab Technical University, Bathinda \\ E-mail: munishcse@gmail.com (Corresponding Author)
}

\begin{abstract}
Deep learning techniques are playing an important role in the classification and prediction of diseases. Undoubtedly deep learning has a promising future in the health sector, especially in medical imaging. The popularity of deep learning approaches is because of their ability to handle a large amount of data related to the patients with accuracy, reliability in a short span of time. However, the practitioners may take time in analyzing and generating reports. In this paper, we have proposed a Deep Neural Network-based classification model for Parkinson's disease. Our proposed method is one such good example giving faster and more accurate results for the classification of Parkinson's disease patients with excellent accuracy of $94.87 \%$. Based on the attributes of the dataset of the patient, the model can be used for the identification of Parkinsonism's. We have also compared the results with other existing approaches like Linear Discriminant Analysis, Support Vector Machine, K-Nearest Neighbor, Decision Tree, Classification and Regression Trees, Random Forest, Linear Regression, Logistic Regression, Multi-Layer Perceptron, and Naive Bayes.
\end{abstract}

Keywords: Parkinson's Disease, Deep Neural Networks, Deep Learning, Classification

\section{Introduction}

Deep learning gained momentum in industry and academia is because of its ability to find complicated patterns in massive datasets. Other approaches are heavy and complex in dealing with massive datasets, and some are unable to handle extensive datasets. Before deep learning, machine learning was popular in many applications but, due to the short-comings of machine learning techniques, deep learning gained popularity. In the health care sector, deep learning plays an important role in early and accurate diagnosis of disease and patient classification in a very short time. Deep Learning [1] is very popular in the diagnosis and the prediction of many diseases like diabetes, cancer, Alzheimer's and Parkinsonism, and many more. In recent years many researchers are also working on Parkinson's disease. The reason behind increasing research in Parkinson's disease is due to a large number of patients falling prey to it day by day. Parkinson's Progression Markers Initiative (PPMI) is the organization that provides many datasets of PD patients based on imaging modality. PPMI provides the dataset of MRI and PET images of patients who have Parkinson's disease in the USA. The actual 
cause of Parkinson's disease (PD) is still unknown, but some common factors, like genes, aging, and lifestyle play a major role. Major research done on PD shows that when dopamine level decreases in neurons, it affects the communication of neurons causing PD. Subjects suffering from PD may have some common symptoms like memory loss, tremor, bradykinesia, and weakness. Many studies show that patients suffering from PD have poor concentration power; the patient is not even able to write properly and easily forgets the daily appointments and social gatherings [2][3][4]. In severe cases, Parkinson's patients are not even capable of introducing themselves due to poor memory and weak concentration [5]. Parkinson's Disease (PD) is the most striking Neuro-Degenerative Disease (NDD) [6] in old age people after Alzheimer [7][8][9] and many researchers are working on PD or Parkinsonism [5][10][11][12]. A study on changes in the handwriting of healthy and person suffering from PD is given in [13] as the level of dopamine de- creases, patients find difficulties in walking, speaking, and writing [13]. researchers have applied deep learning on telemonitoring data of PD patients who have difficulties in speaking. People suffering from PD scramble with vocal symptoms and have trouble in the normal production of vocal sounds [14][15]. This condition is known as dysphonia and is a type of voice disorder including functional problems with voice [16][17].

In this manuscript, we have designed a Deep Neural Network-based model for the classification of Parkinson's Disease (PD) patients. Major contributions of the research are as follows:

- We have designed a Deep Neural Network-based model for the classification of Parkinson's patients and healthy persons. The results show that the model can classify cases with an accuracy of $94 \%$.

- The results have been compared with other existing approaches and findings indicate that the accuracy of the proposed model is higher than the other existing approaches.

- The proposed model is capable of achieving higher accuracy in a smaller number of layers, thus reducing the computational overhead. The Rest of the paper is organized as follows; Background is discussed in Section 2. Related Works are described in Section 3; Methodology is given in Section 4. Experiments and Results are described in Section 5, and Section 6 concludes the manuscript. 


\section{Background}

Machine learning techniques [18] have become increasingly popular due to their capability of giving accurate results in problems such as classification and clustering. A broad classification of different Machine learning techniques is given in Figure 1.

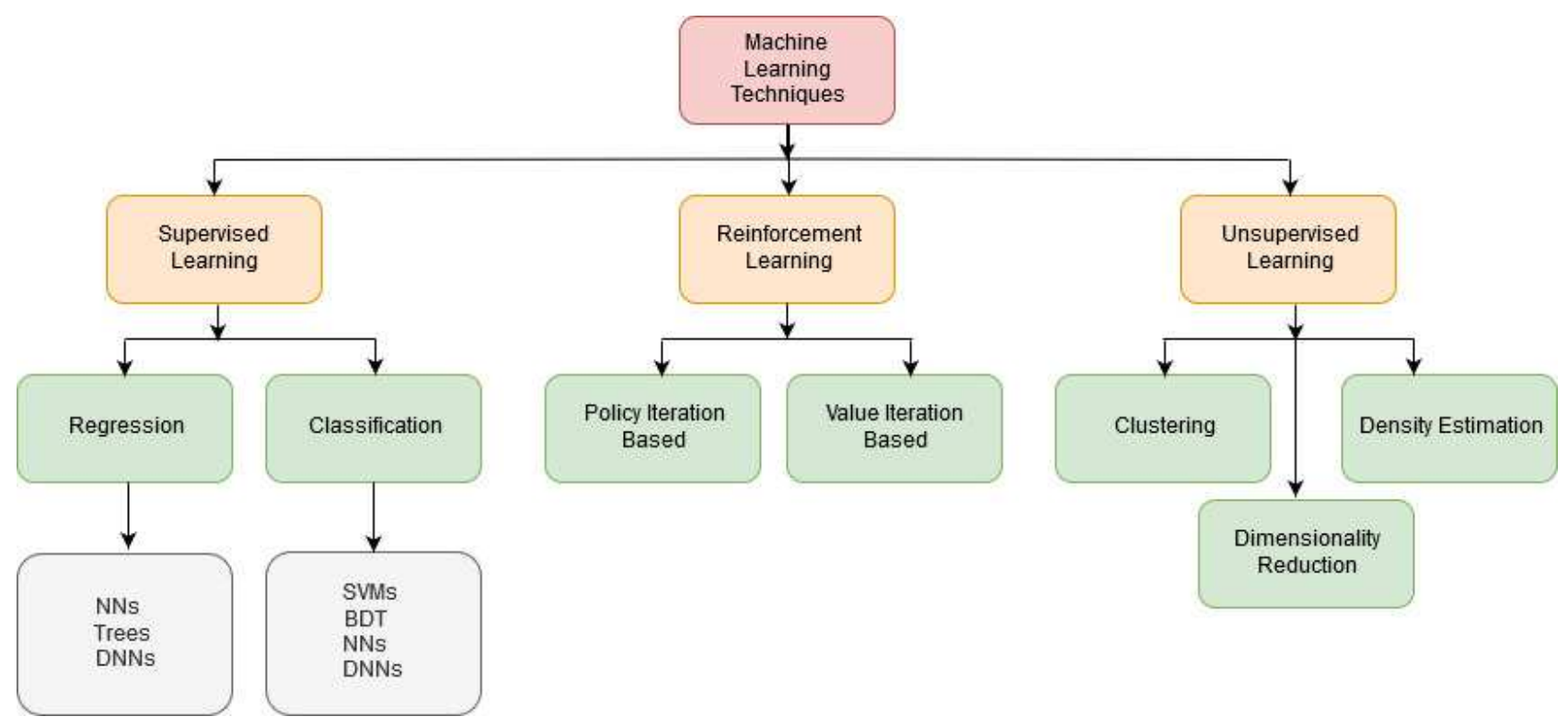

Fig. 1: Broad classification of Machine Learning techniques

Abbreviations used in Figure 1 can be read as NN: Neural Networks, SVM: Support Vector Machine, DNN: Deep Neural Networks BDT: Binary Decision Tree. Among the Machine learning techniques illustrated in Figure 1, Deep learning is an upcoming trend, especially in the health care sector [19][20] [21][22]. Today, various DL-based computer vision applications are performing even better than humans. One such application is identifying indicators for cancer in blood and tumors in MRI scans. DNNs are extensively used in applications like object detection, speech recognition, face recognition, and medical imaging due to their unparalleled results. A DNN can necessarily learn all possible mappings after effective training and make quick and smart predictions, e.g., interpolations and extrapolations for unseen cases. Thus, DL is leaving an impact in the fields of computer vision and medical image processing. A similar impact of DL is seen in domains like voice and text recognition.

\section{Related Works}

Das used different techniques [14] for the prediction of Parkinson's disease. In his study, he found that the overall accuracy of neural networks is $92.9 \%$. There are other physiological problems associated with Parkinson's disease like bradykinesia [17] including slower movement, muscle rigidity, resting tremor, and weakness. This study includes symptoms of PD and the role of surgery and deep brain stimulation. Some researchers are also working on a clinical dataset of PD [23]. They 
developed a deep learning-based FP-CIT SPECT interpretation system which helps in enhancing the imaging diagnosis of PD. This study focused on SWEDD (Scan without Evidence of Dopaminergic Deficit). It helps in differentiating between mild PD patients and severe PD with the help of SPECT imaging giving a total $98.8 \%$ classification accuracy. Many researchers have done a worldwide PD based survey in different countries [15][24]. In this study, they have shown the demographic distribution of the population suffering from PD and the most affected age group. They found that people in the age group of 60 or more are most affected by Parkinson's disease.

Fargel et al. [25] performed a survey of PD patients and neurologists. Out of 500 patients who were surveyed, 49\% were at an early stage and 51\% were suffering from severe PD. This survey focused on the treatment of PD and improving the quality of life of patients. Jankovic [26] discussed the features of PD and their diagnostic methods. Measures towards improving the condition of PD patients have also been discussed. Saeed et al. [27] have described the clinical and pathological features of PD and how structural MRI helps in the diagnosis of PD. Many researchers and academicians are working on different techniques that can be used for the correct classification of Parkinson's disease. Some of the researches done in this area are summed up in Table 1.

Table 1: Related Works on Parkinson's Disease

\begin{tabular}{|c|c|c|c|c|}
\hline Research & $\begin{array}{l}\text { Number of } \\
\text { Features }\end{array}$ & Method(s) & $\begin{array}{l}\text { Performance } \\
\text { Evaluator }\end{array}$ & Major Findings \\
\hline Das [14] & 20 & $\begin{array}{l}\text { NN, DM Neural, } \\
\text { Regression and } \\
\text { DT }\end{array}$ & ROC & $\begin{array}{c}\text { Classification rates are } \\
\text { respectively: } \\
92.9 \%, 84.3 \%, 88.6 \%, 84.3 \%\end{array}$ \\
\hline $\begin{array}{l}\text { Astrom \& } \\
\text { Koker [28] }\end{array}$ & 10 & $\begin{array}{l}\text { Parallel Neural } \\
\text { Network }\end{array}$ & $\begin{array}{c}\text { Accuracy, MSE \& } \\
\text { AUC }\end{array}$ & $\begin{array}{c}\text { They performed experiments } \\
\text { on } 5 \text { different types of } \\
\text { networks, out of which Max } \\
\text { accuracy was } 89.9 \% \text {, lowest } \\
\text { MSE } 0.077 \% \text { and Max AUC } \\
96.7 \%\end{array}$ \\
\hline $\begin{array}{l}\text { Pan et al. } \\
\text { [29] }\end{array}$ & 6 & MLP, RBN, KNN & Accuracy & $\begin{array}{c}\text { MLP with } 79.25 \%, \text { RBN with } \\
80.13 \% \text { and SVM with } \\
81.14 \% \text { Accuracy }\end{array}$ \\
\hline $\begin{array}{l}\text { Mandal \& } \\
\text { Sairam [30] }\end{array}$ & 22 & $\begin{array}{l}\text { SVM, SMO, RF, } \\
\text { Ada Boost \& NN }\end{array}$ & $\begin{array}{l}\text { Accuracy and } \\
\text { precision }\end{array}$ & $\begin{array}{c}\text { Accuracy of the methods are } \\
\text { respectively: } \\
96.58 \%, 95.22 \%, 96.07 \% \\
96.41 \%, 95.05 \%\end{array}$ \\
\hline $\begin{array}{c}\text { Tiwari et al. } \\
\text { [31] }\end{array}$ & 20 & $\begin{array}{c}\text { Bagging, } \\
\text { Boosting, RF, } \\
\text { Rotation Forest, } \\
\text { Random Subspace, }\end{array}$ & $\begin{array}{l}\text { Accuracy, } \\
\text { Precision and } \\
\text { ROC }\end{array}$ & $\begin{array}{c}\text { The accuracy of the methods } \\
\text { are respectively: } 87.7 \%, 91.3 \% \\
, 90.3 \%, 93.3 \%, 90.3 \%, 79 \% \\
88.7 \%, 85.6 \%\end{array}$ \\
\hline
\end{tabular}




\begin{tabular}{|c|c|c|c|c|}
\hline & & $\begin{array}{l}\text { SVM, MLP and } \\
\text { DT }\end{array}$ & & \\
\hline $\begin{array}{l}\text { Nilakshi et } \\
\text { al. [32] }\end{array}$ & 16 & $\begin{array}{l}\text { PCA-NN, PCA- } \\
\text { ANFIS, PCA- } \\
\text { SVR, EM- PCA- } \\
\text { ANFIS, EM- } \\
\text { PCA-SVR }\end{array}$ & MAE & $\begin{array}{l}\text { MAE of Total UP- DRS } \\
\text { respectively: } \\
84 \%, 63.4 \%, 59.9 \%, 53.2 \% \text { and } \\
44.4 \%\end{array}$ \\
\hline $\begin{array}{l}\text { Grover } \\
\text { et al. }[13]\end{array}$ & 16 & DNN & Accuracy & $\begin{array}{c}\text { Accuracy } 81.66 \% \text { for Motor } \\
\text { UPDRS score }\end{array}$ \\
\hline $\begin{array}{l}\text { Gao et al. } \\
\quad[33]\end{array}$ & 129 & $\begin{array}{l}\text { RF, LR, SVM, } \\
\text { NN, XGBoost }\end{array}$ & Accuracy & $\begin{array}{c}\text { Accuracy of RF, LR, SVM, } \\
\text { NN, XGBoost are } 76 \%, \\
43 \%, 74 \%, 65 \%, 73 \% \\
\text { respectively }\end{array}$ \\
\hline $\begin{array}{l}\text { Wrong et } \\
\text { al.[12] }\end{array}$ & - & RF, ANN and DT & $\begin{array}{l}\text { Accuracy, F1 } \\
\text { Score and recall }\end{array}$ & $\begin{array}{c}\text { Accuracy of RF } 83 \% \text { and } \\
\text { recall } 62 \%, \text { ANN recall } 82 \% \\
\text { f1-Score } 78 \% \text { and DT } \\
\text { accuracy } 75 \% \text { and poor re- call } \\
\text { value of } 46 \%\end{array}$ \\
\hline $\begin{array}{l}\text { Gupta et } \\
\text { al.[34] }\end{array}$ & 17 & $\begin{array}{l}\text { KNN \& DT using } \\
\text { OCFA }\end{array}$ & Accuracy & $\begin{array}{l}92.19 \% \text { accuracy with } \\
\text { different datasets and number } \\
\text { of features are also different }\end{array}$ \\
\hline $\begin{array}{l}\text { Mostafa et } \\
\text { al. [11] }\end{array}$ & - & $\begin{array}{l}\mathrm{DT}, \mathrm{NV}, \mathrm{NN}, \mathrm{RF} \\
\text { and SVM }\end{array}$ & Accuracy & $\begin{array}{l}86.44 \% \text { for DT, } 74.11 \% \text { for } \\
\mathrm{NV}, 87.75 \% \text { for } \mathrm{NN}, 86.73 \% \\
\text { for RF and } 86.29 \% \text { for } \mathrm{SVM}\end{array}$ \\
\hline
\end{tabular}

Abbreviations used in Table 1 can be read as RF: Random Forest, NN: Neural Networks, ANN: Artificial Neural Networks, SVM: Support Vector Machine, DT: Decision Tree, PCA-NN: Principal Component Analysis-Neural Networks, PCA-ANFIS: Principal Component Analysis-Adaptive Neuro-Fuzzy Inference System, PCA-SVR: Principal Component Analysis-Support Vector Regression, DNN: Deep Neural Networks, MLP: Multi-Layer Perceptron, ROC: Receiver Operating Characteristic, AUC: Area Under the Curve and OCFA: Optimized cattle fish algorithm. Due to the limitations of existing approaches, we have proposed a Deep Neural Network-based model for the classification of Parkinson's disease. In our proposed model we have tried to increase the classification accuracy and at the same time reduce the over-head of classification by optimizing the number of layers in the model. This model can be further used for the early diagnosis of Parkinson's disease.

\section{Methodology}

In this section, we discuss the proposed Parkinson's disease Classification using the Deep Neural Networks model (PDCD) which uses DNN's for the classification of PD. 


\subsection{Dataset Description}

We have collected the Parkinson's telemonitoring data from the web- site www.kaggle.com available with the name Parkinson Disease Prediction. The data-set has multivariate attribute characteristics, and the number of at- tributes is 21 . The status column specifies whether the subject is suffering from PD or not. The database contains a voice record of 42 patients at an early stage of PD. These 42 people were recruited for 6 months trial on a telemonitoring device for remote symptom progression monitoring. Brief description of the attributes in the dataset are given in Table-2.

Table 2: Dataset Description

\begin{tabular}{|l|l|}
\hline Name of Attribute & Description \\
\hline MDVP:Fo(Hz) & Average vocal fundamental frequency \\
\hline MDVP:Fhi(Hz) & Maximum vocal fundamental frequency \\
\hline $\begin{array}{l}\text { Jitter(\%), Jitter(Abs), Jitter:RAP, Jitter:PPQ5, } \\
\text { Jitter:DDP }\end{array}$ & $\begin{array}{l}\text { Several measurements of variation in } \\
\text { fundamental frequency }\end{array}$ \\
\hline $\begin{array}{l}\text { Shimmer, Shimmer(db), Shimmer:APQ3, } \\
\text { Shimmer:APQ5, Shimmer:APQ11, } \\
\text { Shimmer:DDA } \\
\text { NHR and HNR }\end{array}$ & $\begin{array}{l}\text { Several measurements of variation in frequency } \\
\text { amplitude }\end{array}$ \\
\hline RPDE \& D2 & $\begin{array}{l}\text { Two measures of ratio of noise to tonal com- } \\
\text { ponents in the voice }\end{array}$ \\
\hline DFA & A non-linear dynamical complexity measure \\
\hline Spread1, Spread2 \& PPE & $\begin{array}{l}\text { Signal fractal scaling exponent } \\
\text { Non-linear measure of fundamental frequency } \\
\text { variation }\end{array}$ \\
\hline Status & $\begin{array}{l}\text { Health Status of Patient, if it is one then } \\
\text { Parkinson, otherwise zero indicates healthy }\end{array}$ \\
\hline
\end{tabular}

\subsection{Proposed Algorithm}

In this sub-section, we discuss the proposed Algorithm that classifies the subject with Status 1 indicating that the subject is suffering from Parkinson's and Status 0 indicating that the subject is Healthy.

Table 3: Notations used in Algorithm 1

\begin{tabular}{|l|l|}
\hline $\mathrm{N}$ & Features in dataset \\
\hline DataTR & Training Data \\
\hline DataTest & Testing Data \\
\hline $\mathrm{N}_{\mathrm{L}}$ & Number of Layers \\
\hline Accuracymax & Maximum accuracy of model \\
\hline Accuracy & Classification accuracy of model \\
\hline
\end{tabular}




\section{Algorithm 1 Algorithm of Parkinson's Neural Networks (PDCD) model}

Input: $\mathrm{N}$ (21 features from the dataset)

Output: Status $1=$ Parkinson s Disease \& Status $0=$ Healthy

Accuracy: $=0$

Accuracy $_{\text {max }}:=0$

DataTR $:=0.75 \times \mathrm{N}$

DataT est $:=0.25 \times \mathrm{N}$

$\mathrm{N}_{\mathrm{L}}:=100$

while $\mathrm{i}=1$ to $\mathrm{N}_{\mathrm{L}}$ do

TrainModelPDCD(DataTR,Status)

TestModelPDCD(DataTest,Status)

Accuracy $:=$ CalculateAccuracyofthemodel

if Accuracy $>$ Accuracy $_{\max }$ then

Accuracy $_{\text {max }}:=$ Accuracy

end if

if Status $==1$ then

return Parkinson's-Disease

else

return Healthy

end if

end while

We have designed a Deep Neural Network (DNN) based model for the classification of PD. The broad layout of the PDCD model is shown in Figure 2. 


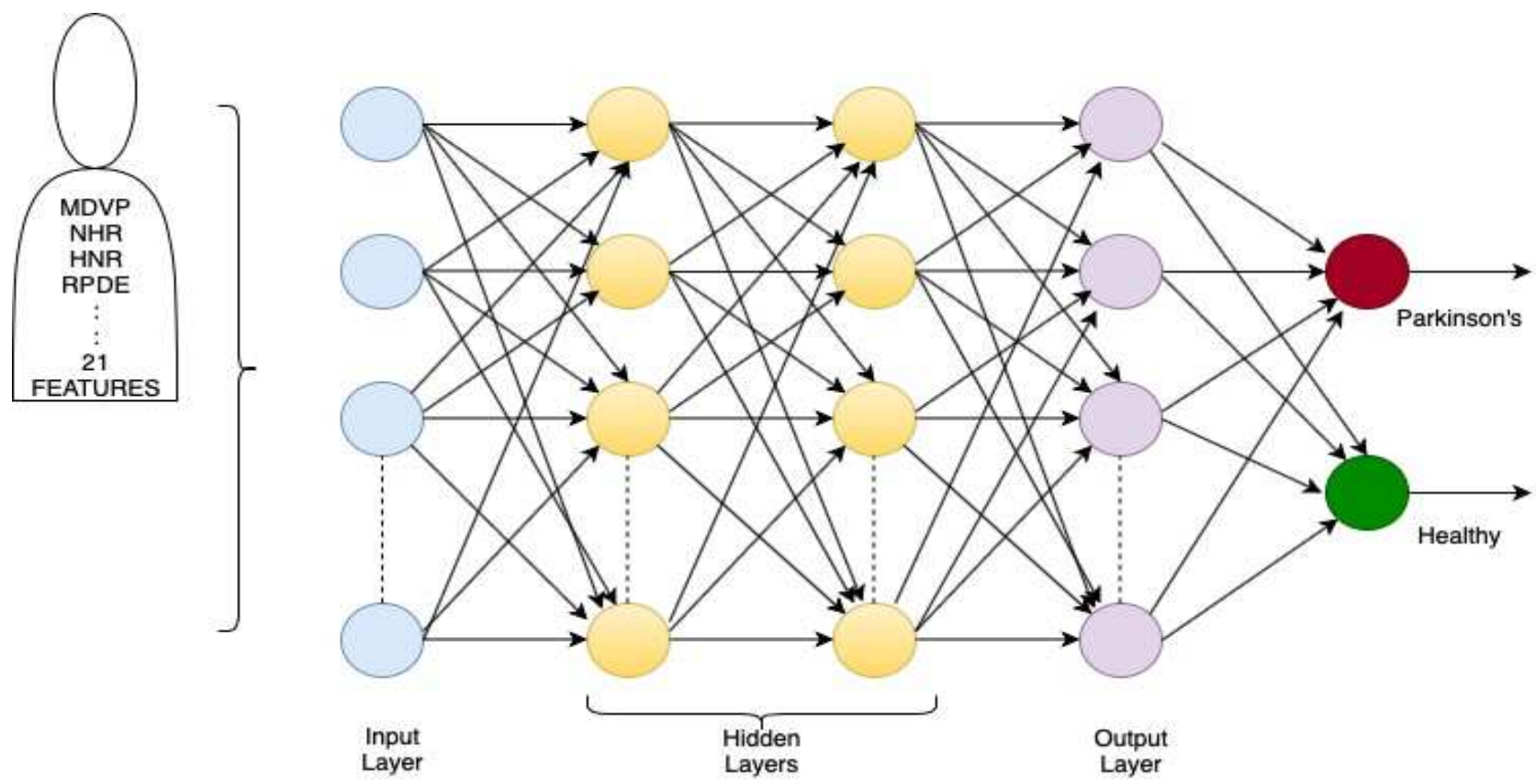

Fig. 2: Classification of Parkinson's Disease using DNN

We performed the data pre-processing, and after the selection of features, we chose 21 features for the classification of PD. Our model consists of total of 11 layers, in which the Input Layer takes 21 features as input and gives output in the next layer containing 42 nodes for processing. The first layer has 924 parameters. The Layer-wise distribution of the nodes and the respective parameters are given in Table 4.

Table 4: Proposed Model Summary

\begin{tabular}{|l|l|l|}
\hline Layer (Type) & Output Shape & Parameters \\
\hline Dense_1(Dense) & (None ,42) & 924 \\
\hline Dense_2(Dense) & (None ,84) & 3612 \\
\hline Dense_3(Dense) & (None ,168) & 14280 \\
\hline Dense_4(Dense) & (None ,200) & 33800 \\
\hline Dense_5(Dense) & (None ,100) & 20100 \\
\hline Dense_6(Dense) & (None ,84) & 8484 \\
\hline Dense_7(Dense) & (None ,42) & 3570 \\
\hline Dense_8(Dense) & (None ,21) & 903 \\
\hline Dense_9(Dense) & (None ,10) & 220 \\
\hline $\begin{array}{l}\text { Dense_10(Dense) } \\
\text { Dense_11(Dense) }\end{array}$ & (None ,5) & 55 \\
\hline
\end{tabular}


The model was tested on different parameters as illustrated in Table 4; it gave the best results for 11 Layers. If the number of Layers was increased beyond this, there was no significant improvement in the results. In some cases, there was a drop in classification accuracy. The experiments were executed for 1000 simulations and after using the proposed PDCD model, the highest accuracy achieved was $94.87 \%$. Detailed results are discussed in the next section.

\section{Experiments and Results}

\subsection{Data Pre-processing}

All the features were taken during the pre-processing step, and we performed some basic checking for the null values or missing values. In our dataset, there were no null or missing values. So, we did not use any missing value handling techniques during the pre-processing step. We normalized the data, after splitting it into training and testing datasets. We used Standard Scaler to avoid manual processing. After data pre-processing the PDCD model was applied to the data and the results are discussed in the next subsection.

\subsection{Model Optimisation}

Optimization is an important task when we are dealing with datasets. We used "adam" optimizer for optimizing the model which had some features like, ease of implementation, computationally efficient for processing, takes less memory, is appropriate for handling noisy and null data, and typically demands negligible tuning for handling.

\subsection{Classification}

Classification is the last step before fitting the model and evaluating the model. After that, we classify it into 2 categories Parkinson's disease and Healthy. In our dataset, status 0 indicates a healthy person and 1 is for Parkinson's patients. As shown in Table 4 for value 0 in the status column we get precision $82 \%$, recall $90 \%$, F1 score $86 \%$ and support is 10 while for value 1 in the status column we get precision $96 \%$, recall 93\%, F1 score 95\% and support 29. Total precision of the classification model is $93 \%$, recall is $92 \%$, the F1-Score is $92 \%$ and support is 39 and overall classification accuracy is $94 \%$ which is better as compared to other methods. 
Table 5: Classification results of proposed model.

\begin{tabular}{|c|c|c|c|c|}
\hline Status & Precision & Recall & F1- Score & Support \\
\hline 0 & $82 \%$ & $90 \%$ & $86 \%$ & 10 \\
\hline 1 & $96 \%$ & $93 \%$ & $95 \%$ & 29 \\
\hline Total & $93 \%$ & $92 \%$ & $92 \%$ & 39 \\
\hline
\end{tabular}

\subsection{Workflow of the proposed work}

Figure 3 illustrates the complete workflow of the proposed work. The details are discussed in the next sub-sections.

\subsection{Performance comparison of PDCD model with other approaches}

The proposed model was compared with other well established classification techniques [35] like KNearest Neighbour (KNN) [34], Support Vector Machine (SVM) [29][30][31][33], Linear Discriminant Analysis (LDA) [36], Decision Tree (DT) [37][38], Classification and Regression Tree (CART) [39], Random Forest (RF) [37], Linear Regression (LR) [39], Logistic Regression (LogR) [39], Multi-Layer Perceptron (MLP) [29][31], Naive Bayes (NB) [40] Comparative analysis is shown in Table 6.

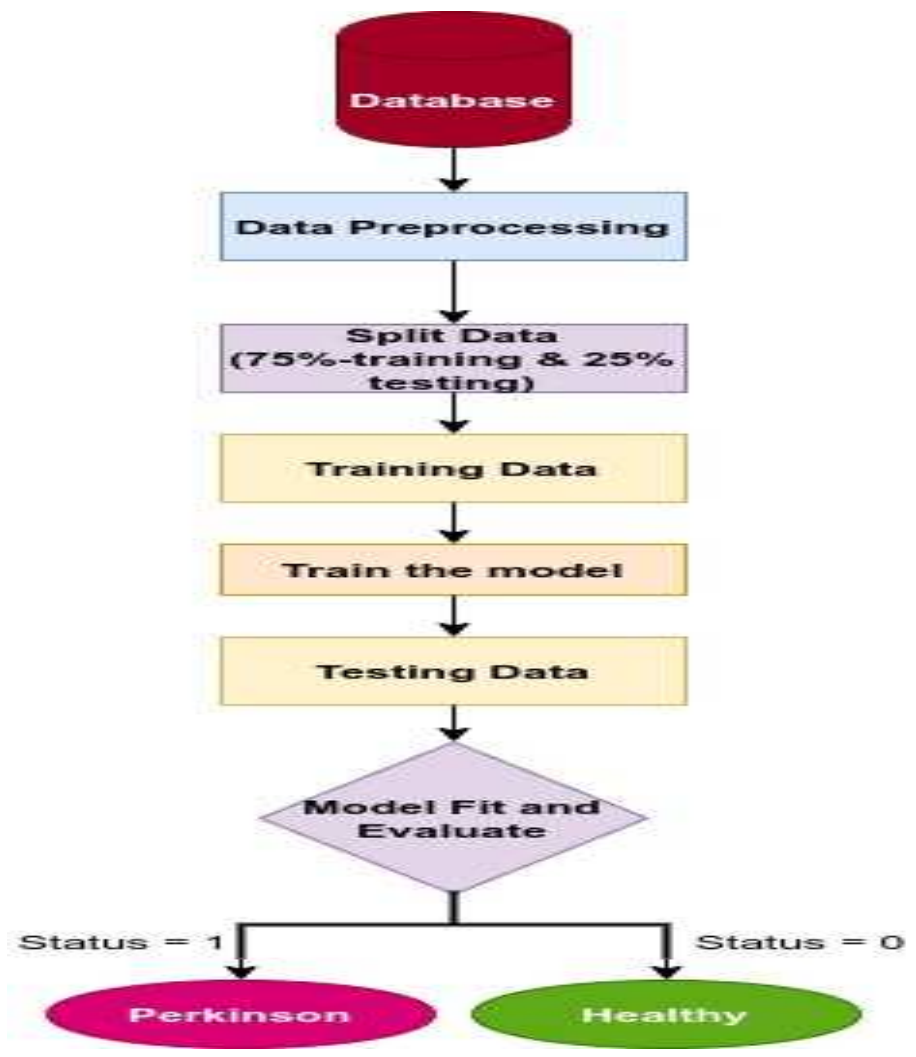

Fig. 3: Proposed work flow 
Table 6: Comparative analysis of proposed PDCD model with other methods.

\begin{tabular}{|c|c|c|c|c|}
\hline Methods & Accuracy & Precision & Recall & F1-Score \\
\hline KNN & $89 \%$ & $91 \%$ & $90 \%$ & $89 \%$ \\
\hline SVM & $74 \%$ & $74 \%$ & $74 \%$ & $74 \%$ \\
\hline LDA & $89 \%$ & $90 \%$ & $90 \%$ & $89 \%$ \\
\hline DT & $79 \%$ & $81 \%$ & $79 \%$ & $80 \%$ \\
\hline CART & $85 \%$ & $85 \%$ & $86 \%$ & $85 \%$ \\
\hline RF & $85 \%$ & $88 \%$ & $86 \%$ & $84 \%$ \\
\hline LR & $89 \%$ & $90 \%$ & $90 \%$ & $89 \%$ \\
\hline Log R & $66 \%$ & $72 \%$ & $67 \%$ & $68 \%$ \\
\hline MLP & $91 \%$ & $93 \%$ & $92 \%$ & $91 \%$ \\
\hline NB & $65 \%$ & $87 \%$ & $65 \%$ & $68 \%$ \\
\hline PDCD & $\mathbf{9 4 \%}$ & $\mathbf{9 3 \%}$ & $\mathbf{9 2 \%}$ & $\mathbf{9 2 \%}$ \\
\hline
\end{tabular}

Abbreviations used in Table 6 can be read as KNN: K-Nearest Neighbour, SVM: Support Vector Machine and, LDA: Linear Discriminant Analysis, DT: Decision Tree, CART: Classification and Regression Tree, RF: Random Forest, LR: Linear Regression, LogR: Logistic Regression, MLP: Multi-Layer Perceptron, NB: Naive Bayes. As shown in Table 6, our proposed model has the highest accuracy of $94 \%$ and MLP achieves the second-highest accuracy after our proposed method. The precision of our proposed method is highest among all the methods. MLP, KNN, and LDA also achieve good precision. Similarly, recall of our proposed methods is highest and MLP also achieves recall equal to our proposed method.

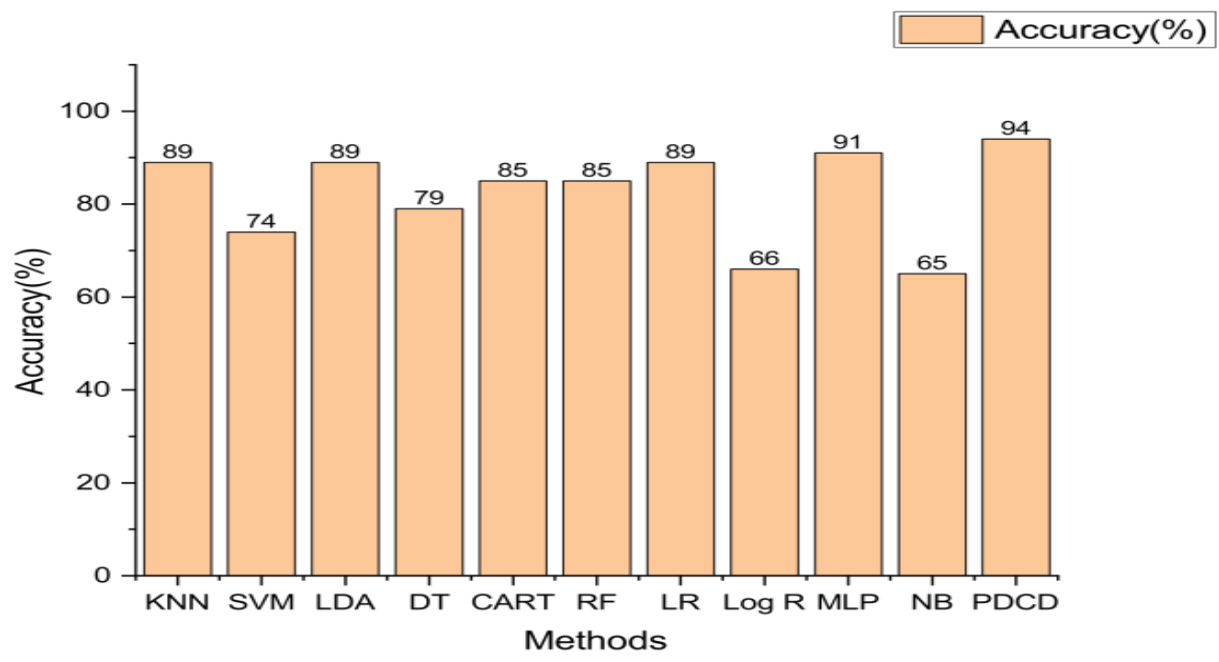

Fig. 4: Methods V/S Accuracy 
As shown in Figure 4 the accuracy of the proposed method PDCD is the highest among all the methods MLP, KNN and LDA are also having good accuracy rate and NB has the least accuracy.

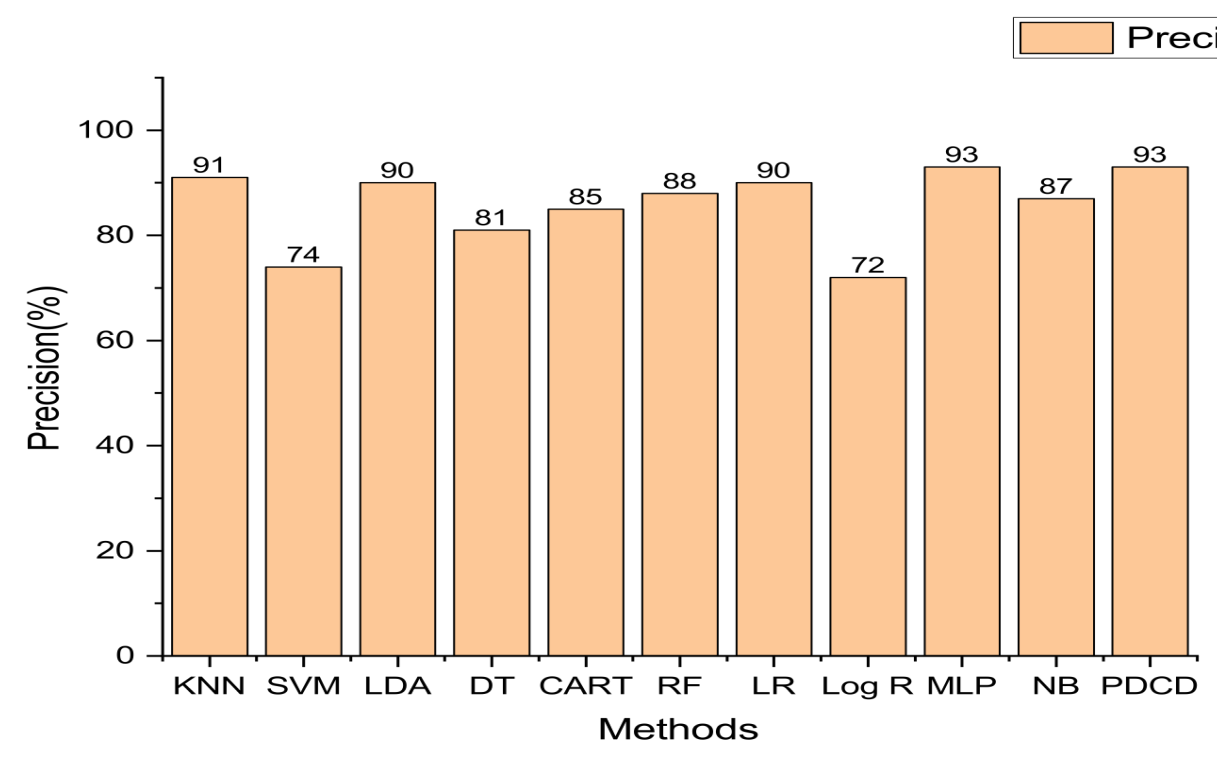

Figure 5: Methods V/S Precision

A comparative analysis of precision is shown in Figure 5. The precision of the proposed method PDCD is the highest among all the methods MLP, LR, KNN, and LDA also show good precision rate and Logistic regression has the least precision.

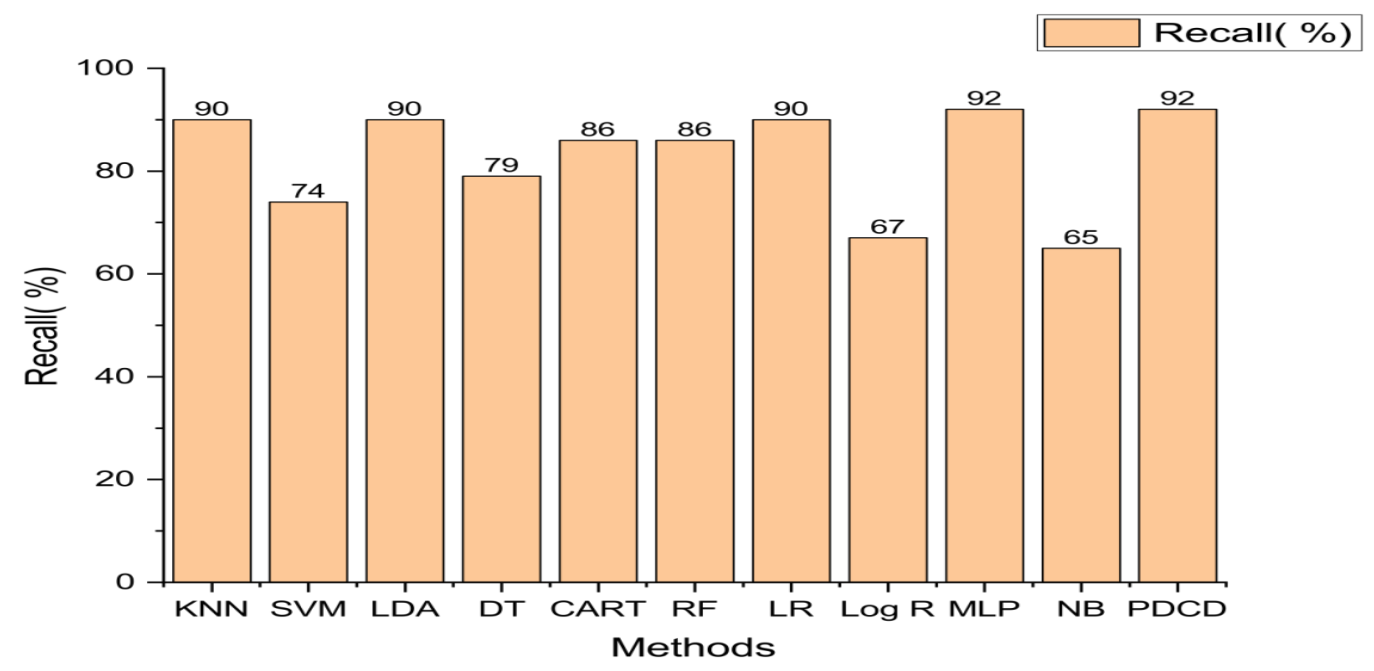

Figure 6: Methods V/S Recall

Figure 6 Shows that the Recall of the proposed method is the highest among all the methods MLP, KNN and LDA also perform well in achieving precision and NB has the least Recall value. 


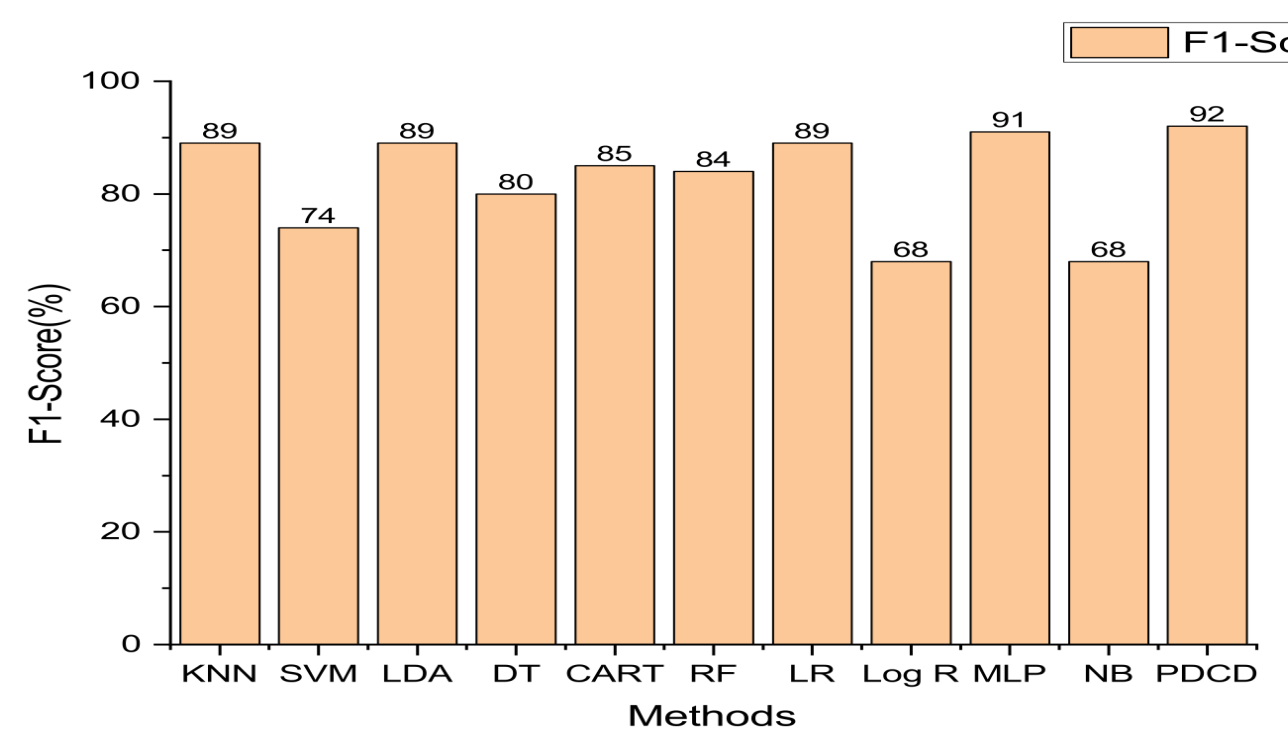

Figure 7: Methods V/S F1-Score

Figure 7 shows the F1-Score of the proposed method PDCD is the highest among all the methods. MLP, KNN, LR, and LDA also have relatively good F1-Score and NB and LogR have the least F1Score.

\subsection{Effect of different parameters on model}

Table 7: Effect of different parameters on model and its performance

\begin{tabular}{|c|c|c|c|c|}
\hline $\begin{array}{c}\text { Number of } \\
\text { layers }\end{array}$ & $\begin{array}{c}\text { Number of } \\
\text { nodes }\end{array}$ & Parameters & Epochs & $\begin{array}{c}\text { Accuracy } \\
(\%)\end{array}$ \\
\hline 3 & 13 & 181 & 10 & 74.35 \\
\hline 3 & 19 & 229 & 20 & 92.30 \\
\hline 3 & 53 & 1365 & 50 & 84.61 \\
\hline 3 & 64 & 1849 & 30 & 79.48 \\
\hline 3 & 48 & 1145 & 40 & 74.35 \\
\hline 4 & 25 & 271 & 10 & 74.35 \\
\hline 4 & 25 & 271 & 20 & 89.74 \\
\hline 4 & 50 & 1154 & 30 & 74.35 \\
\hline 4 & 69 & 1943 & 40 & 89.74 \\
\hline 5 & 112 & 2736 & 20 & 84.61 \\
\hline 5 & 112 & 2736 & 30 & 87.17 \\
\hline 5 & 97 & 3185 & 30 & 74.35 \\
\hline & & & & \\
\hline
\end{tabular}




\begin{tabular}{|c|c|c|c|c|}
\hline 5 & 139 & 5411 & 40 & 74.35 \\
\hline 5 & 150 & 6368 & 50 & 92.30 \\
8 & 416 & 33990 & 20 & 87.17 \\
\hline 8 & 426 & 28739 & 10 & 74.35 \\
\hline 8 & 364 & 27098 & 30 & 87.17 \\
\hline 8 & 394 & 27098 & 40 & 74.35 \\
\hline 8 & 394 & 33990 & 20 & 87.17 \\
\hline 10 & 920 & 147300 & 30 & 97.48 \\
\hline 10 & 920 & 147300 & 50 & 87.17 \\
\hline 10 & 636 & 64048 & 10 & 74.35 \\
\hline 10 & 694 & 78142 & 20 & 74.35 \\
\hline 11 & 757 & 85954 & 100 & 94.87 \\
\hline 11 & 778 & 85954 & 10 & 74.35 \\
\hline 11 & 757 & 85261 & 100 & 92.30 \\
\hline 11 & 799 & 87710 & 50 & 92.30 \\
\hline
\end{tabular}

We performed many trials with different types of parameters in the model. After performing the trials, we conclude that an increment in the number of layers enhances the accuracy of the model. The effect of different parameters on the performance of the model is given in Table 6. We tried to experiment with the model up to 100 layers but there was no significant improvement in the accuracy of the model after 11 layers. Thus, we used 11layers in all the experiments. Some of the observations regarding the accuracy of the proposed model are: As numbers of layers are increased the number of nodes also increases and the accuracy of the model also increases. If we increase the number of layers and simultaneously increase the number of epochs, then the accuracy of the model increases. If there is increase in the number of layers then number of parameters also increases which also increases the accuracy of the model. Let the number of layers in the model be $\mathrm{N}_{\mathrm{L}}$, the number of nodes is denoted by $\mathrm{N}_{\text {Nodes, }}$ the number of parameters of nodes denoted by $\mathrm{P}$, and number of epochs be denoted by $\mathrm{E}$. The relationship between different parameters is as shown in Equations 1, 2, 3 and 4. Here accuracy indicates the accuracy of the model.

Many trials with different types of parameters were performed by us and the conclusion we reached was that whenever there was an increment in the number of layers, it enhances the accuracy of the model. Different parameters affect the performance affected the performance of the model which are 
detail in Table 7. Whatever accuracy that we explode observed was up-till 11 layers through the trials of model execution were made up to 100 layers. Therefore, we use only 11 layers in the experiments there was some observation regarding the accuracy of the proposed model the increase in the number of the layers is directly proportional to the number of the nodes and the accuracy of the model. If we increase the number of layers then the number of parameters also increases which also increases the accuracy of the model. Let the number of layers in the model be $\mathrm{N}_{\mathrm{L}}$, number of nodes be denoted by $\mathrm{N}_{\text {Nodes}}$, number of parameters of nodes denoted by $\mathrm{P}$ and number of epochs be denoted by $\mathrm{E}$.

The relationships between different parameters are as shown in Equations 1, 2, 3, and 4. Here accuracy indicates the accuracy of the model.

$$
\begin{aligned}
& \mathrm{N}_{\mathrm{L}} \propto \text { Accuracy } \\
& \mathrm{N}_{\text {Nodes }} \propto \text { Accuracy } \\
& \mathrm{P} \propto \text { Accuracy } \\
& \mathrm{E} \propto \text { Accuracy }
\end{aligned}
$$

\section{Conclusions}

Deep learning has a promising future in the engineering and health sector. In this paper, we designed a Deep Neural Network-based model for the classification of PD. The proposed model has an accuracy of $94.87 \%$ which is reasonably good as compared to other classification techniques. Different parameters were tested and the results indicate that Deep Neural Networks can be effectively used for early diagnosis of the disease.

\section{Compliance with Ethical Standards}

Research involving Human Participants and/or Animals: No human and animal participants were used.

Funding Information: This research is funded by UGC under the scheme RGNF-SC funded by the Ministry of social justice empowerment and ministry of tribal affairs of India, New Delhi, India.

\section{Conflict of interest: None}

\section{Authorship contributions}

Monika Jyotiyana: Conceptualization, Formal analysis, Data curation, Writing - original draft

Nishtha Kesswani: Project administration, Formal analysis, Resources, Writing - original draft, Supervision, Writing - review \& editing 
Munish Kumar: review \& editing, Supervision, Project administration

\section{Références}

[1] M. Jyotiyana, N. Kesswani, Deep learning and the future of biomedical image analysis, in: Deep Learning Techniques for Biomedical and Health Informatics, Springer, 2020, pp. 329345 .

[2] L. Hartelius, P. Svensson, Speech and swallowing symptoms associated with parkinson's disease and multiple sclerosis: a survey, Folia phoni- atrica et logopaedica 46 (1994) 9-17.

[3] M. Politis, Neuroimaging in parkinson disease: from research setting to clinical practice, Nature Reviews Neurology 10 (2014) 708.

[4] M. B. Stern, A. Siderowf, Parkinson's at risk syndrome: can parkinson's disease be predicted?, Movement disorders 25 (2010) S89-S93.

[5] M. Grossman, A. Cooke, C. DeVita, C. Lee, D. Alsop, J. e. Detre, J. Gee, W. Chen, M. Stern, H. Hurtig, Grammatical and resource components of sentence processing in parkinson's disease: An fmri study, Neurology 60 (2003) 775-781.

[6] M. Jyotiyana, N. Kesswani, A study on deep learning in neurodegener- ative diseases and other brain disorders, in: Rising Threats in Expert Applications and Solutions, Springer, pp. 791-799.

[7] S.-J. Son, J. Kim, H. Park, Structural and functional connectional fin- gerprints in mild cognitive impairment and alzheimer's disease patients, PloS one 12 (2017) e0173426.

[8] S. Kl“oppel, C. M. Stonnington, C. Chu, B. Draganski, R. I. Scahill, J. D. Rohrer, N. C. Fox, C. R. Jack Jr, J. Ashburner, R. S. Frackowiak, Automatic classification of mr scans in alzheimer's disease, Brain 131 (2008) 681-689.

[9] C. R. Munteanu, C. Fernandez-Lozano, V. M. Abad, S. P. Ferna' ndez, J. A lvarez-Linera, J. A. Herna ndez-Tamames, A. Pazos, Classification of mild cognitive impairment and alzheimer's disease with machine- learning techniques using $1 \mathrm{~h}$ magnetic resonance spectroscopy data, Ex- pert Systems with Applications 42 (2015) 6205-6214.

[10] R. Prashanth, S. D. Roy, P. K. Mandal, S. Ghosh, Automatic classifica- tion and prediction models for early parkinson's disease diagnosis from spect imaging, Expert Systems with Applications 41 (2014) 3333-3342. 
[11] S. A. Mostafa, A. Mustapha, M. A. Mohammed, R. I. Hamed, N. Arunk- umar, M. K. A. Ghani, M. M. Jaber, S. H. Khaleefah, Examining multiple feature evaluation and classification methods for improving the diagnosis of parkinson's disease, Cognitive Systems Research 54 (2019) 90-99.

[12] T. J. Wroge, Y. Ö̈kanca, C. Demiroglu, D. Si, D. C. Atkins, R. H. Ghomi, Parkinson's disease diagnosis using machine learning and voice, in: 2018 IEEE Signal Processing in Medicine and Biology Symposium (SPMB), IEEE, 2018, pp. 1-7.

[13] S. Grover, S. Bhartia, A. Yadav, K. Seeja, et al., Predicting severity of parkinson's disease using deep learning, Procedia computer science 132 (2018) 1788-1794.

[14] R. Das, A comparison of multiple classification methods for diagnosis of parkinson disease, Expert Systems with Applications 37 (2010) 1568-1572.

[15] M. d. De Rijk, C. Tzourio, M. Breteler, J. Dartigues, L. Amaducci, S. Lopez-Pousa, J. Manubens-Bertran, A. Alperovitch, W. A. Rocca, Prevalence of parkinsonism and parkinson's disease in europe: the eu- roparkinson collaborative study. european community concerted action on the epidemiology of parkinson's disease., Journal of Neurology, Neurosurgery \& Psychiatry 62 (1997) 10-15.

[16] A. K. Ho, R. Iansek, C. Marigliani, J. L. Bradshaw, S. Gates, Speech impairment in a large sample of patients with parkinson's disease, Be- havioural neurology 11 (1999) 131-137.

[17] A. Berardelli, J. Rothwell, P. Thompson, M. Hallett, Pathophysiology of bradykinesia in parkinson's disease, Brain 124 (2001) 2131-2146.

[18] M. Jyotiyana, N. Kesswani, Classification and prediction of alzheimer's disease using multilayer perceptron, International Journal of Reasoning-based Intelligent Systems 12 (2020) $255-263$.

[19] M. I. Razzak, S. Naz, A. Zaib, Deep learning for medical image process- ing: Overview, challenges and the future, in: Classification in BioApps, Springer, 2018, pp. 323-350.

[20] L. Deng, G. Hinton, B. Kingsbury, New types of deep neural network learning for speech recognition and related applications: An overview, in: 2013 IEEE International Conference on Acoustics, Speech and Signal Processing, 2013, pp. 8599-8603.

[21] D. Rav`1, C. Wong, F. Deligianni, M. Berthelot, J. Andreu-Perez, B. Lo, G.-Z. Yang, Deep learning for health informatics, IEEE journal of biomedical and health informatics 21 (2016) 4-21. 
[22] J.-G. Lee, S. Jun, Y.-W. Cho, H. Lee, G. B. Kim, J. B. Seo, N. Kim, Deep learning in medical imaging: general overview, Korean journal of radiology 18 (2017) 570-584.

[23] H. Choi, S. Ha, H. J. Im, S. H. Paek, D. S. Lee, Refining diagnosis of parkinson's disease with deep learning-based interpretation of dopamine transporter imaging, NeuroImage: Clinical 16 (2017) 586-594.

[24] Z.-X. Zhang, G. C. Rom' an, Worldwide occurrence of parkinson's disease: an updated review, Neuroepidemiology 12 (1993) 195-208.

[25] M. Fargel, B. Grobe, E. Oesterle, C. Hastedt, M. Rupp, Treatment of parkinson's disease, Clinical drug investigation 27 (2007) 207-218.

[26] J. Jankovic, Parkinson's disease: clinical features and diagnosis, Journal of neurology, neurosurgery \& psychiatry 79 (2008) 368-376.

[27] U. Saeed, J. Compagnone, R. I. Aviv, A. P. Strafella, S. E. Black, A. E. Lang, M. Masellis, Imaging biomarkers in parkinson's disease and parkinsonian syndromes: current and emerging concepts, Translational neurodegeneration 6 (2017) 8.

[28] F. Astro $\dddot{m}$, R. Koker, A parallel neural network approach to prediction of parkinson's disease, Expert systems with applications 38 (2011) 12470-12474.

[29] S. Pan, S. Iplikci, K. Warwick, T. Z. Aziz, Parkinson's disease tremor classification-a comparison between support vector machines and neural networks, Expert Systems with Applications 39 (2012) 10764-10771.

[30] I. Mandal, N. Sairam, New machine-learning algorithms for prediction of parkinson's disease, International Journal of Systems Science 45 (2014) 647-666.

[31] A. K. Tiwari, Machine learning based approaches for prediction of parkinson's disease, Mach Learn Appl 3 (2016) 33-39.

[32] M. Nilashi, O. Ibrahim, A. Ahani, Accuracy improvement for predicting parkinson's disease progression, Scientific reports 6 (2016) 1-18.

[33] C. Gao, H. Sun, T. Wang, M. Tang, N. I. Bohnen, M. L. Mu`1ler, T. Her- man, N. Giladi, A. Kalinin, C. Spino, et al., Model-based and model-free machine learning techniques for diagnostic prediction and classification of clinical outcomes in parkinson's disease, Scientific reports 8 (2018) 1-21. 
[34] D. Gupta, A. Julka, S. Jain, T. Aggarwal, A. Khanna, N. Arunkumar, V. H. C. de Albuquerque, Optimized cuttlefish algorithm for diagnosis of parkinson's disease, Cognitive systems research 52 (2018) 36-48.

[35] J. Han, J. Pei, M. Kamber, Data mining: concepts and techniques, Elsevier, 2011.

[36] Z. A. Dastgheib, B. Lithgow, Z. Moussavi, Diagnosis of parkinson's disease using electrovestibulography, Medical \& biological engineering \& computing 50 (2012) 483-491.

[37] K. Oppedal, T. Eftestøl, K. Engan, M. K. Beyer, D. Aarsland, Clas- sifying dementia using local binary patterns from different regions in magnetic resonance images, Journal of Biomedical Imaging 2015 (2015) 5.

[38] G. Yadav, Y. Kumar, G. Sahoo, Predication of parkinson's disease using data mining methods: A comparative analysis of tree, statistical and support vector machine classifiers, in: 2012 National Conference on Computing and Communication Systems, IEEE, 2012, pp. 18.

[39] R. G. Ramani, G. Sivagami, Parkinson disease classification using data mining algorithms, International journal of computer applications 32 (2011) 17-22.

[40] D. A. Morales, Y. Vives-Gilabert, B. Go'mez-Ans'on, E. Bengoetxea, P. Larran aga, C. Bielza, J. Pagonabarraga, J. Kulisevsky, I. Corcuera- Solano, M. Delfino, Predicting dementia development in parkinson's disease using bayesian network classifiers, Psychiatry Research: NeuroImaging 213 (2013) 92-98. 